\title{
Biodentine: A Unique Bio-Active Endodontic Material with Versatile Uses
}

\author{
I Anand Sherwood $^{1 *}$, James L Gutmann ${ }^{2}$, Geeth Deepika ${ }^{1}$, Vanitha Sadashivam ${ }^{1}$, Evangelin $\mathbf{J}^{1}$, Divya \\ Meena ${ }^{1}$, Nivedha $V^{1}$, Ramyadharshini $T^{1}$, Joyson Joe Asir ${ }^{1}$, Subashri ${ }^{1}$, CT Valliappan ${ }^{1}$, Pavula $S^{1}$ \\ ${ }^{1}$ Department of Conservative Dentistry and Endodontics, CSI College of Dental Sciences and Research, \\ Madurai, Tamil Nadu, India
}

${ }^{2}$ Professor and Chair, Department of Endodontics and Postgraduate Program Director in Endodontics at Nova Southeastern University College of Dental Medicine, Florida, United States

*Corresponding Author: I Anand Sherwood, Department of Conservative Dentistry and Endodontics, CSI College of Dental Sciences and Research, Madurai, Tamil Nadu, India; Email: anand.sherwood@ gmail.com

Received Date: 22-07-2020; Accepted Date: 14-08-2020; Published Date: 21-08-2020

Copyright $^{\odot} 2020$ by Sherwood AI, et al. All rights reserved. This is an open access article distributed under the terms of the Creative Commons Attribution License, which permits unrestricted use, distribution, and reproduction in any medium, provided the original author and source are credited.

\begin{abstract}
Biodentine is bioactive endodontic cement. This case series report contains some common clinical indications where Biodentine been used successfully and some off-label usage of this material, which has enabled successful clinical outcomes. Cases present in the report are from the year 2016 to the present date.
\end{abstract}

\section{Keywords}

Apexification; Bioactive Endodontic Cement; Biodentine; Open Apex; Perforation Repair; Periapical Pathology; Resorption

\section{Introduction}

Biodentine is a "bioactive endodontic cement", which implies the release of calcium ions, electroconductivity, production of calcium hydroxide, formation of interfacial layer between the cement and the cementum / dentinal wall, and formation of apatite crystals over the surface of material in a synthetic tissue fluid environment such as phosphate buffer solution [1,2]. Most of the bioactive endodontic cements available in market are either calcium silicate or calcium

Sherwood AI | Volume 1; Issue 2 (2020) | JDHOR-1(2)-011 | Review Article 
aluminate-based [1]. Biodentine is a tri-calcium silicate containing cement with the unique characteristics of rapid setting, development of early high strength, and a higher reactivity than other calcium silicates [1]. Biodentine is presented as powder/liquid system comprising a powder of tri-calcium silicate, di-calcium silicate, calcium carbonate, $\mathrm{CaO}$ and zirconium oxide as radio pacifier. Liquid consists of calcium chloride and a hydro soluble polymer. Biodentine was developed as a multipurpose, dentin and root replacement material and has been shown to exhibit excellent biocompatibility properties $[3,4]$.

This case series report contains some common clinical indications where Biodentine been used successfully and some off-label usage of this material, which has enabled successful clinical outcomes. Cases present in the report are from the year 2016 to the present date.

\section{Clinical Cases}

\section{Perforation Management}

Two patients with complaints of pain in relation to root canal treated teeth reported to department in years 2010 and 2011. On radiograph examination indicated there was a perforation and missed canal (Fig. 1). The missed canal was treated and obturated with guttapercha and zinc oxide eugenol sealer. The perforation was sealed with glass ionomer cement; this material has been shown to demonstrate inferior outcomes compared to bioactive cements in perforation sealing.

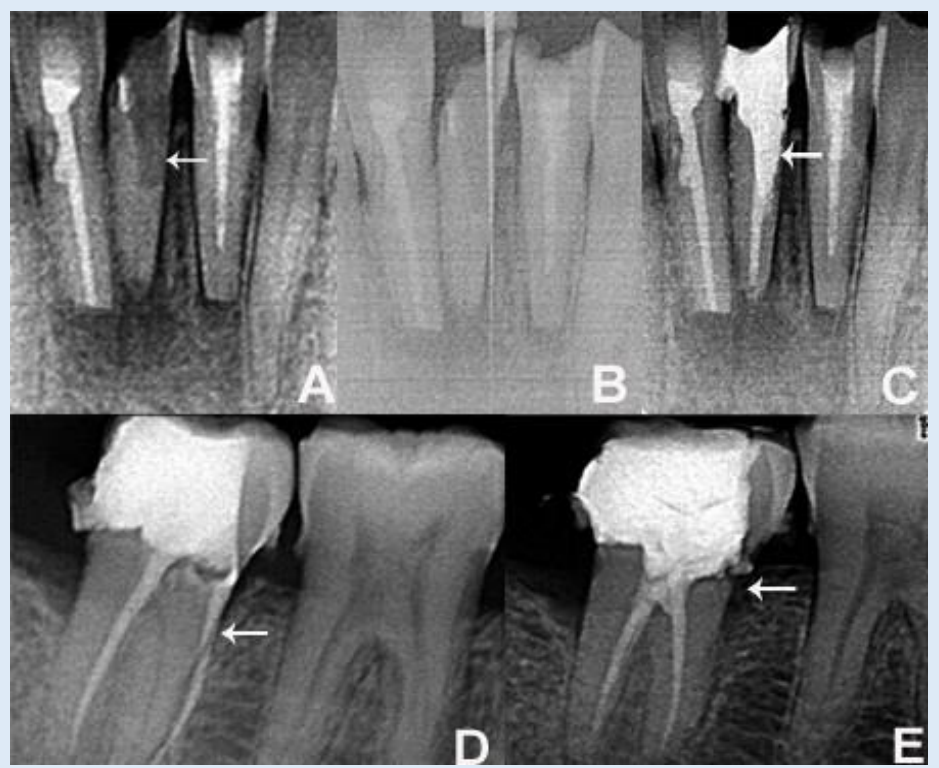

Figure 1: A, B, C: Mandibular central incisor perforation management with glass ionomer cement. Arrows point to area of perforation. D, E: Mandibular second molar perforation in the mesial root managed with glass ionomer cement. Arrows point to area of perforation.

Sherwood AI | Volume 1; Issue 2 (2020) | JDHOR-1(2)-011 | Review Article

Citation: Sherwood AI, et al. Biodentine: A Unique Bio-Active Endodontic Material with Versatile

Uses. J Dental Health Oral Res. 2020;1(2):1-15. 
In 2016, a 45 yr-old female patient with pain in relation to an attempted root canal procedure on the right maxillary first molar presented for treatment. The periapical radiograph showed a perforation in close proximity to the mesio-buccal canal orifice (Fig. 2). Perforation management was done using an operating microscope; placing Biodentine in more precise manner.

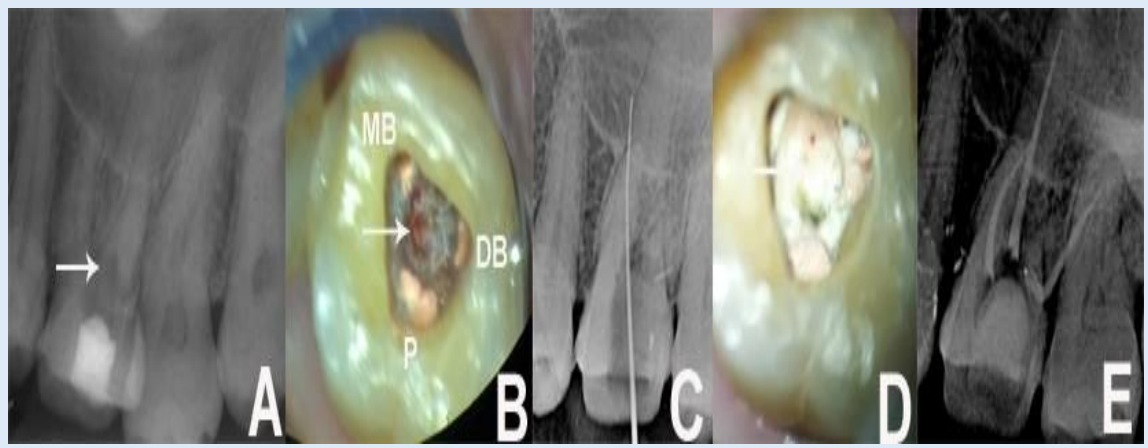

Figure 2: A: Pre-operative radiograph of right maxillary first molar arrows point to perforation close to mesio-buccal (MB) root. B: Under operating microscope magnification the perforation (arrow) is located. C: Root canal file placed in the perforation. D: Perforation sealed with Biodentine (arrow). E: Post-obturation periapical radiograph.

In 2019, a female patient aged 40 years reported with complaints of pain in relation to root canal treated left mandibular first and second molar and periapical radiographic examination showed 3 major errors; a perforation, root canal instrument separation and gutta percha points beyond apex in both the molar teeth (Fig. 3A). Root canal retreatment and perforation management with Biodentine was completed using an operating microscope followed by a proper coronal restoration (Fig. 3A i-x1).

A 20 yr-old male patient reported for post-endodontic restoration for root canal treated left mandibular first molar teeth, for which there was significant loss of tooth structure. A fiber post placement was recommended in the distal canal of tooth (Fig. 3B). During post space preparation a strip perforation occurred, which was managed with Biodentine using an operating microscope followed by an endocrown as post-endodontic restoration (Fig. 3B iivii). 


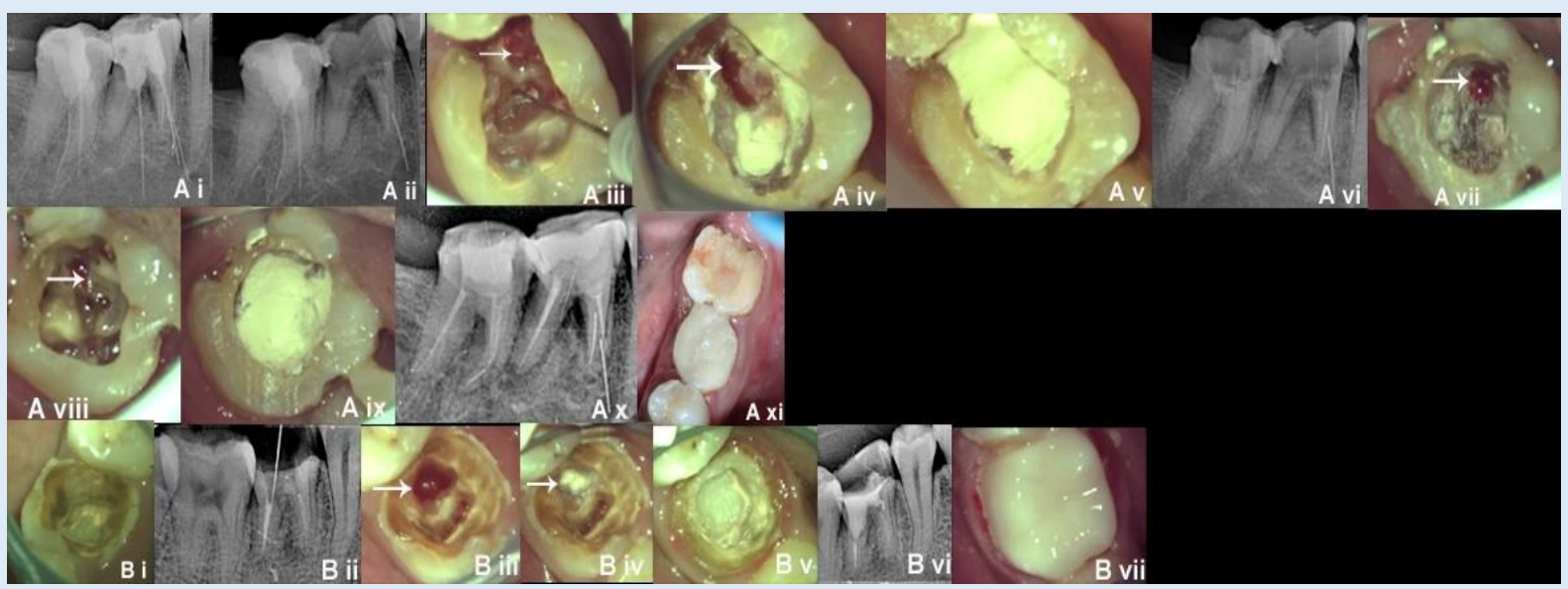

Figure 3: Ai: Pre-operative radiograph of inadequately root canal treated left mandibular first and second molar with instrument separation and gutta percha points beyond apex. Aii: Gutta percha point removed from the left mandibular first molar. Aii: Perforation (arrow) detected near the distal canal of the left mandibular first molar under opierating microscope. Aiv: Perforation (arrow) after obturation of the root canals in the mandibular first molar. Av: Perforation and root canal orifices sealed with Biodentine. Avi. Periapical radiograph showing removal of gutta percha points from mandibular first and second molar. Avii: Perforation (arrow) detected close to the distal canal of the left mandibular second molar. Aviii: Perforation (arrow) after obturation of the root canals in left mandibular second molar.

Aix: Perforation and root canal orifices sealed with Biodentine. Ax: Post-obturation radiograph. Axi: Post-endodontic restoration of the left mandibular first and second molar. Bi: Root canal treated left mandibular first molar tooth with significant tooth structure loss. Bii: Periapical radiograph showing post space preparation in the distal canal and file placed in the strip perforation. Biii: Perforation (arrow) in the distal canal under operating microscope. Biv:. Perforation sealed with Biodentine. Bv: Endocrown preparation of the left mandibular first molar. Bvi: Radiograph demonstrating the perforation seal with Biodentine. Bvii: Lithium di silicate endocrown placed in the left mandibular first molar tooth.

Misdirection during access preparation resulting in a perforation is a common risk associated with Pulp Canal Obliterated (PCO) traumatized maxillary incisor teeth. In 2017, a 35 yr-old male patient presented with complaints of pain and discoloured maxillary incisors. Radiographic examination indicated there was a misdirected access preparation in the maxillary left central incisor that resulted in a perforation (Fig. 4). On Cone Beam Computed Tomography (CBCT) scan examination the precise location of the perforation was assessed. Canal was traced by extending the access preparation using ultrasonic diamond abrasive tips and the canal was obturated with gutta-percha and sealer. The perforation was closed with Biodentine and the tooth was restored with a full coverage ceramic crown. Two-year posttreatment $\mathrm{CBCT}$ and radiograph images show no evidence of alveolar bone loss and excellent periapical healing (Fig. 4G,H). 


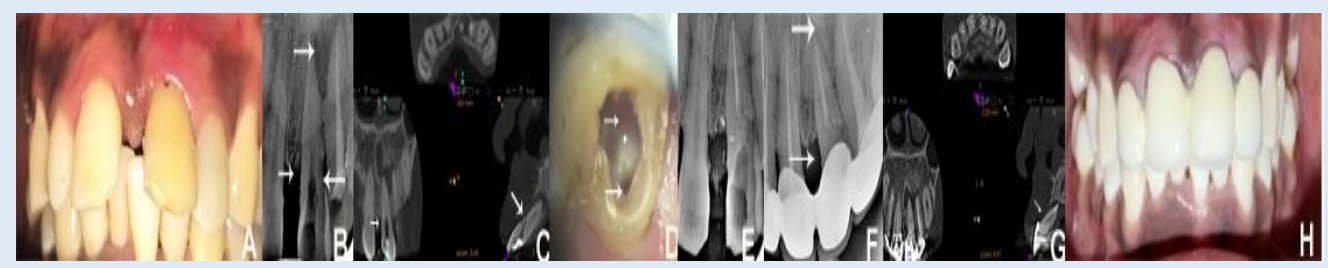

Figure 4: A: Pre-operative photograph of left maxillary central incisor with discoloration due to Pulp Canal Obliteration (PCO) and labial proclination, spacing. B: Pre-operative radiograph showing alveolar crestal bone level (arrow), perforation (arrow) and canal obiteration (arrow). C: CBCT image showing alveolar bone level (arrow) and perforation (arrow). D: Access preparation of the left maxillary central incisor under operating microscope showing perforation (arrow) and canalorifice (arrow). E: Post-obturation radiograph with Biodentine sealing of the perforation. F: Two year post-treatment radiograph demonstrating intact alveolar crestal bone height (arrow) and excellent periapical healing (arrow). G: Two year post-treatment CBCT image shows intact alveolar crestal bone height

(arrow) in the left maxillary central incisor. H: Full coverage ceramic crowns placed to correct the labial proclination and spacing from left maxillary lateral incisor to right maxillary lateral incisor.

\section{External Resorption Management}

The use of Biodentine has provided excellent post-treatment results in management of external root resorption due to various etiologies. In 2017, a 46 yr-old female patient presented with complaints of discoloration and pain in a previously traumatized maxillary left central incisor. On radiographic examination there was external cervical resorption on the root, which was confirmed with CBCT (Fig. 5 A). The root canal was obturated with Biodentine as sealer and thermoplasticized gutta-percha. The resorptive defect was restored following a full thickness muco-periosteal flap reflection with resin-modified glass ionomer cement and using Biodentine as a base.

In 2018, a 45 yr-old male patient presented with complaints of pain in traumatized maxillary right central incisor; a periapical radiograph showed a cervical resorptive defect (Fig. 5 B). A CBCT scan detected external resorption in the maxillary right central incisor. The apical onethird of root canal below the resorptive defect was obturated with thermoplasticized guttapercha, using Biodentine as root canal sealer. The resorptive defect was restored with Biodentine as a base and resin modified glass ionomer cement following palatal full thickness muco-periosteal flap reflection (Fig. 5B). The access preparation was restored along with resorptive defect with Biodentine base and resin modified glass ionomer cement.

In 2017, a 52 yr-old female patient reported to the department for root canal treatment in left maxillary second molar tooth. Upon close examination of the periapical radiograph, the distobuccal root showed evidence of resorption, which was confirmed with a CBCT scan (Fig. 5C). The disto-buccal root was obturated with Biodentine cement, while the rest of the root canals

Sherwood AI | Volume 1; Issue 2 (2020) | JDHOR-1(2)-011 | Review Article

Citation: Sherwood AI, et al. Biodentine: A Unique Bio-Active Endodontic Material with Versatile

Uses. J Dental Health Oral Res. 2020;1(2):1-15.

DOI: http://dx.doi.org/10.46889/JDHOR.2020.1206 
were obturated with a single-cone greater taper gutta-percha cones and zinc oxide eugenol sealer. A two-year recall radiograph showed the root canal treated tooth with no progression of resorption or development of periapical pathosis (Fig. 5C).

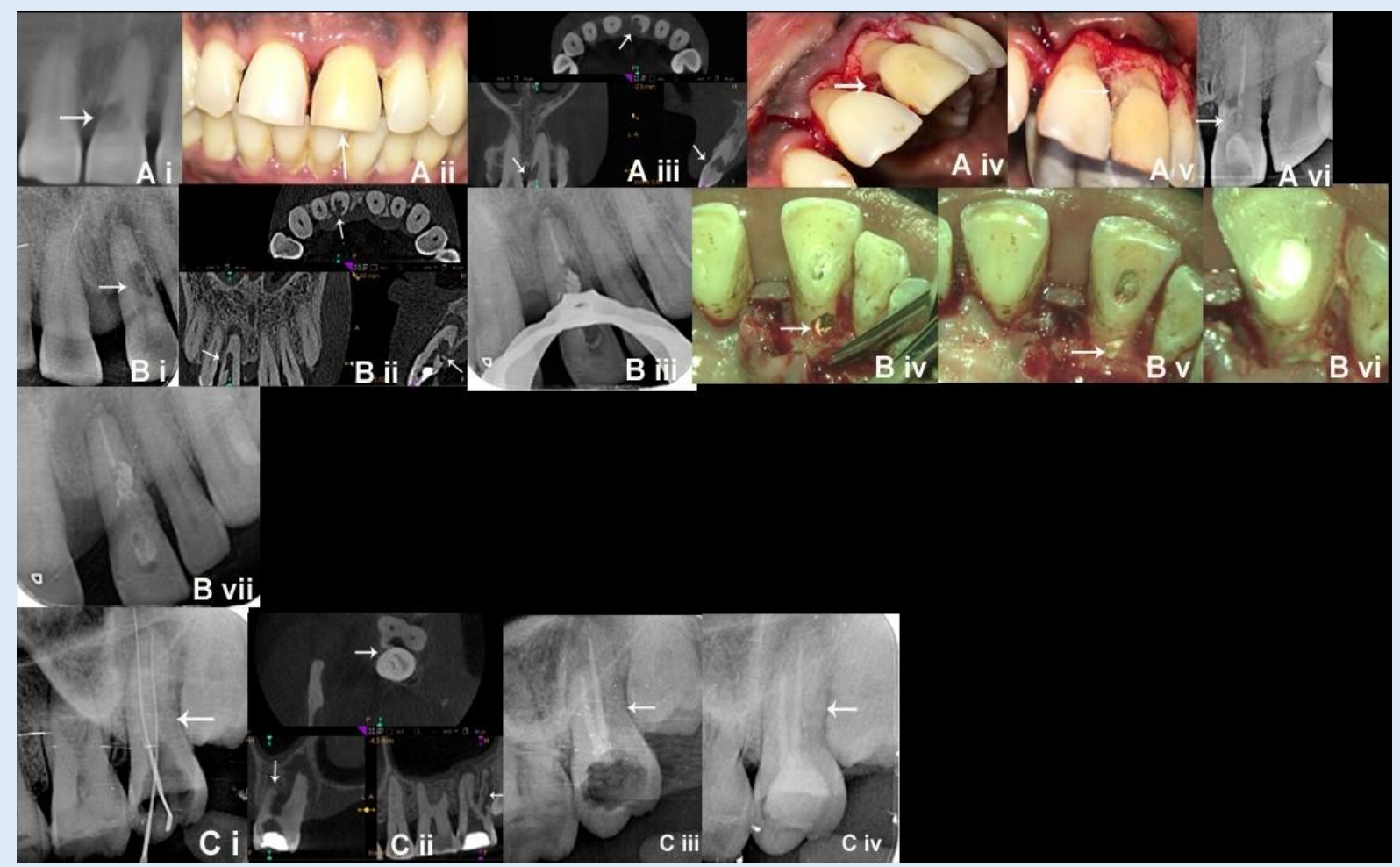

Figure 5: Ai: Pre-operative radiograph of left maxillary central incisor with cervical resorption (arrow). Aii: Pre-treatment photograph of discolored left maxillary central incisor (arrow). Aiii: CBCT image show external cervical resorption (arrow) coronal to the alveolar crestal bone. Aiv: Labial full thickness muco-periosteal flap reflected to access the resorptive defect (arrow) after completion of root canal treatment. Av: Resorptive defect (arrow) sealed

with Biodentine base and resin modified glass ionomer cement. Avi: Post-treatment radiograph showing obturated left maxillary central incisor with resorptive defect sealed (arrow).

Bi: Pre-operating radiograph of maxillary right central incisor with cervical resorption (arrow). Bii: Pre-treatment CBCT scan show external cervical resorptive defect coronal to alveolar crestal bone extending more towards the palatal aspect of right maxillary central incisor. Biii: Radiograph showing root canal apical to resorptive defect obturated with thermoplasticized gutta percha. Biv: Palatal full thickness muco-periosteal flap reflected to access the resorptive defect (arrow). Bv: Resorptive defect sealed with Biodentine base (arrow). Bvi: Biodentine also placed through the access preparation to allow for better sealing of the cervical resorptive defect. Bvii: Post-treatment radiograph displaying canal obturation and resorptive defect sealing.

Ci: Left maxillary second molar radiograph showing evidence of disto-buccal root resorption (arrow). Cii: Pre-operative CBCT image show external resorption (arrow) in the disto-buccal

Sherwood AI | Volume 1; Issue 2 (2020) | JDHOR-1(2)-011 | Review Article

Citation: Sherwood AI, et al. Biodentine: A Unique Bio-Active Endodontic Material with Versatile

Uses. J Dental Health Oral Res. 2020;1(2):1-15. 
root. Ciii: Post-obturation radiograph mesio-buccal and palatal canal obturation with gutta

percha and disto-buccal root canal filled with Biodentine (arrow). Civ: Two-year recall radiograph displaying excellent healing and no progression of resorption in disto-buccal root (arrow).

\section{Replacement Resorption Management}

In June 2019, a 17 yr-old male patient with pain in a traumatized maxillary right central incisor reported to department. The patient gave a history of replacement of both the maxillary central incisor following avulsion. A periapical radiograph showed complete resorption of maxillary left central incisor root and right central incisor had extensive external replacement resorption approximating the pulp canal space (Fig. 6). Root canal was completed with Biodentine as the obturation material. An 8-month review radiograph and CBCT scan indicated there was no progression of the resorptive defect (Fig. 6).

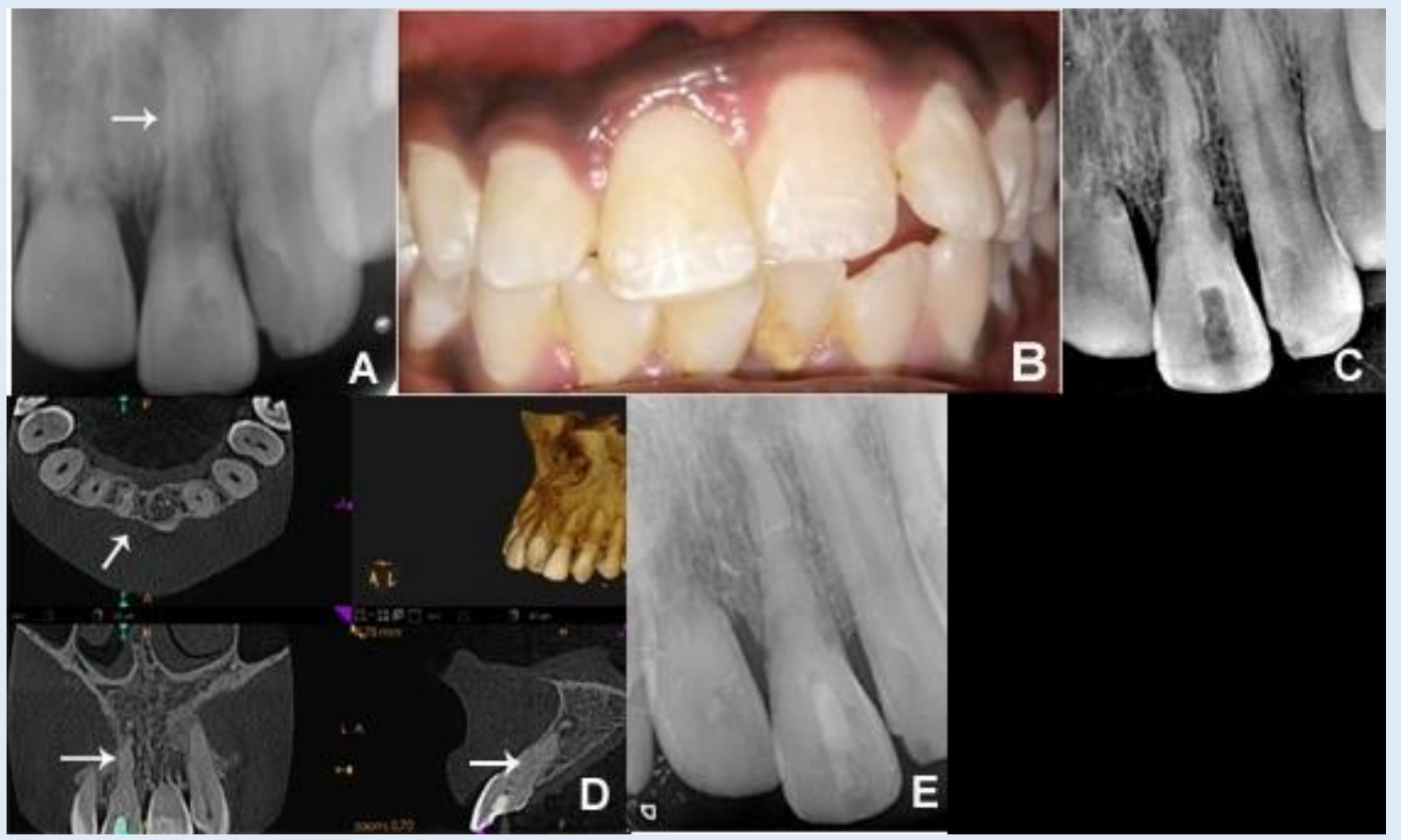

Figure 6: A: Pre-operative radiograph showing extensive replacement resorption (arrow) in the right maxillary central incisor. B: Pre-treatment photograph displaying ankylosed and infra-occlusion position of left maxillary central incisor and labial positioning of right maxillary central incisor. C: Post root canal treated right maxillary central incisor (arrow) canal filled with Biodentine. D: Eight months post-treatment CBCT image displaying nonprogression of the external resorptive defect (arrow). E: Eight months post-treatment radiograph showing the non-progression of the replacement resorption. 


\section{Root Fracture Management}

A 25 yr-old male patient reported to department with complaints of pain in fractured right maxillary central incisor in the year 2017 (Fig. 7). Radiographic examination showed a horizontal root fracture in maxillary left lateral incisor below the alveolar crestal bone. The fractured root segment below the fracture line and alveolar crestal bone was not able to be accessed. The coronal fracture segment had no mobility the pulp chamber and the root canal portion in this segment was cleaned, shaped and obturated with Biodentine cement (Fig. 7). Sixteen months later the patient reported for replacement of the avulsed left maxillary central incisor. A periapical radiograph of maxillary left lateral incisor at this time showed an intact apical root segment with continuous lamina dura and PDL width in normal dimensions (Fig. 7).

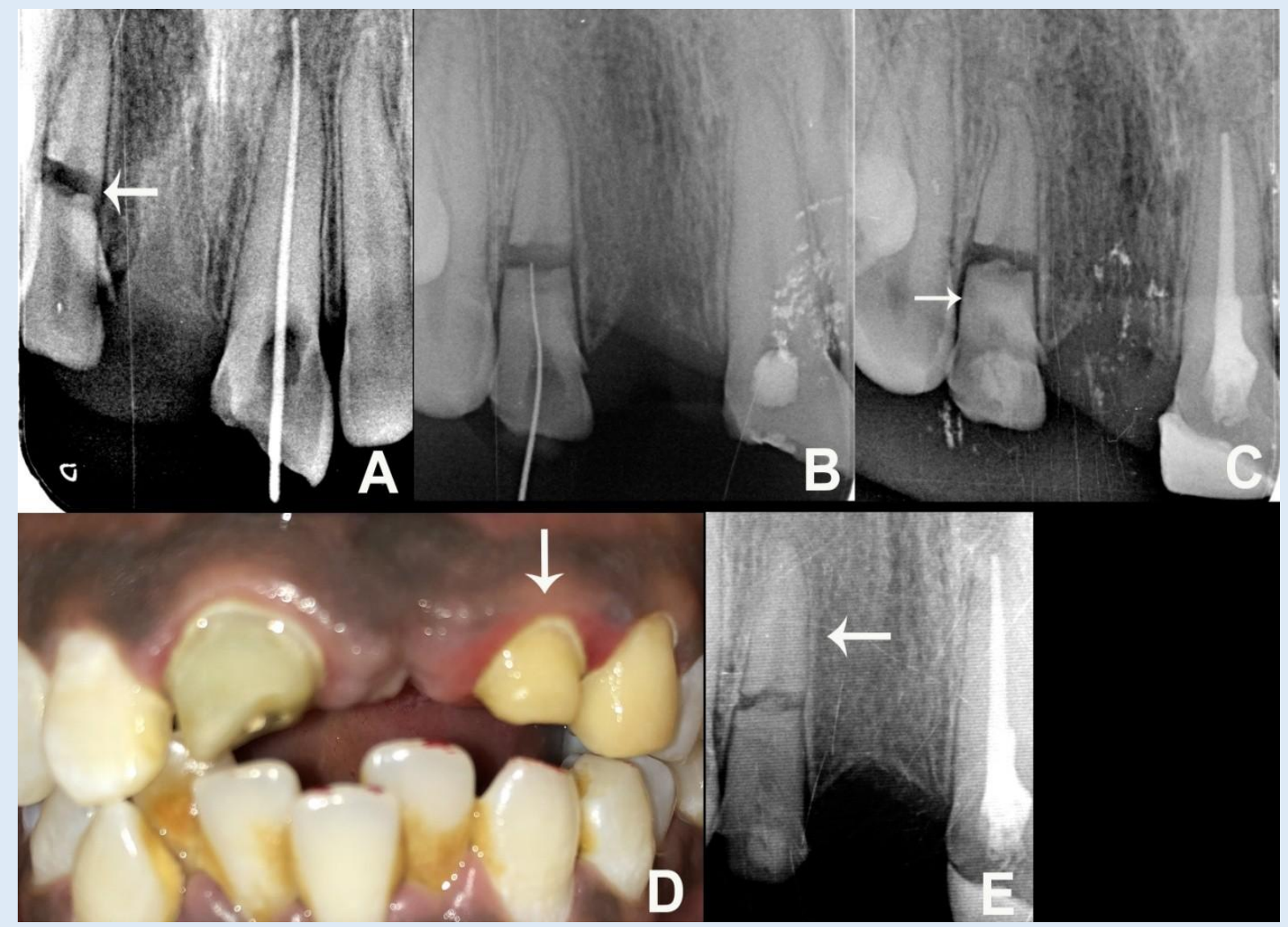

Figure 7: A: Pre-operative radiograph of left maxillary lateral incisor with horizontal root fracture (arrow) below the alveolar bone crest level. B: Coronal segment of the root coronal ot fracture line is cleaned and shaped. C: Coronal segment of root portion of left maxillary lateral incisor (arrow) is filled with Bio-dentine. D: 16-months post-treatment photograph of the left maxillary lateral incisor (arrow). E: 16-months post-treatment radiograph showing intact PDL width and lamina dura in left maxillary lateral incisor (arrow). 


\section{Immature Traumatized Necrotic Pulp (Open Apex) Maxillary Incisor Management}

The management of this procedure and its outcome evaluation are registered with clinical trial registry of India (CTRI/2020/02/023309). The use of Biodentine as obturating material or as apical root canal plug in immature maxillary incisors with necrotic pulps was initiated in 2016. For the first two years of this trial, the entire canal obturation was done with Bio-dentine; beginning in 2018 its use was changed to an apical plug. Armamentarium used for management of apically wide open canals is shown in Fig. 8A, along with its application. In the initial year only the post-treatment outcome was planned for evaluation with CBCT scans (Fig. 8). However, in 2017 a decision was made to assess the pre- and post-operative status of these teeth with CBCT scans (Fig. 8). Thus far, 58 teeth from 54 patients with preoperative CBCT scans have been managed. Root canals of these teeth are minimally instrumented and working length is determined using a phosphate plate sensor; irrigation is done with normal saline solution activated with ultrasonic activation; Triple-Antibiotic Paste (TAP) was used as intracanal medicament for 15 days. Obturation of root canal was completed with a Biodentine apical plug and the canal was back-filled with thermoplasticized gutta-percha. Thirty-five (35) patients have returned for evaluation with $12 \pm 2$ months post-treatment CBCT scans (Fig. 8). Excellent periapical healing was noted in all the post-treatment CBCT scans. The main drawback of this procedure was discoloration of the tooth caused by TAP, but this was reversed with three-sittings of walking bleaching using sodium perborate and $37 \%$ hydrogen peroxide followed by composite restoration or full coverage ceramic crowns (Fig. 9).

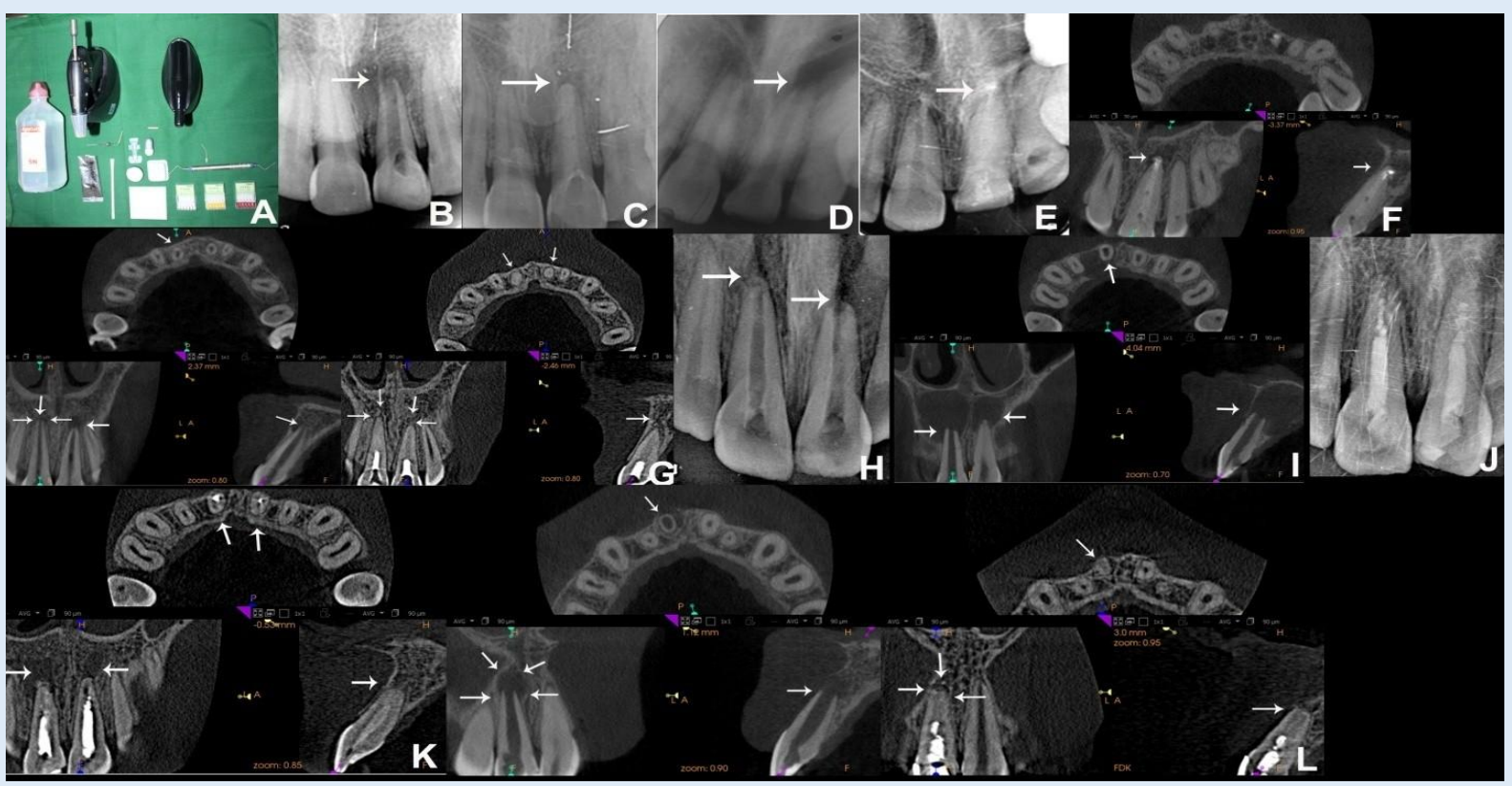

Figure 8: A: Armamentarium used for Biodentine apexification procedure. B, C: Pre-and 9 months post-operative radiograph of right maxillary central incisor with open apex (arrow) 
managed with Biodentine apexification in the year 2016. D, E, F: Pre- and 12-months posttreatment radiograph and CBCT image showing excellent healing (arrow) with Biodentine

apexification in right maxillary central incisorwith open apex (arrow) and periapical radiolucency (arrow) for a 21 yr-old male patient in the year 2016. G: Pre- and 12-months post-treatment CBCT of 14 yr-old female patient with both the maxillary central incisors exhibiting open apex (arrow) and periapical radiolucency (arrow) managed with Biodentine apexification in the year 2017. H, I, J, K: Pre and 12-months post-treatment radiographs and

CBCT images of both maxillary central incisors with open apex (arrow) and periapical radiolucency (arrow) in a 15 yr-old male patient managed with Biodentine apexfication in the year 2018. L: Pre- and 19- months post-treatment CBCT images of right maxillary central incisor with open apex (arrow) and periapical radiolucency (arrow) exhibiting excellent healing in a 15 yr-old female patient in the year 2018.

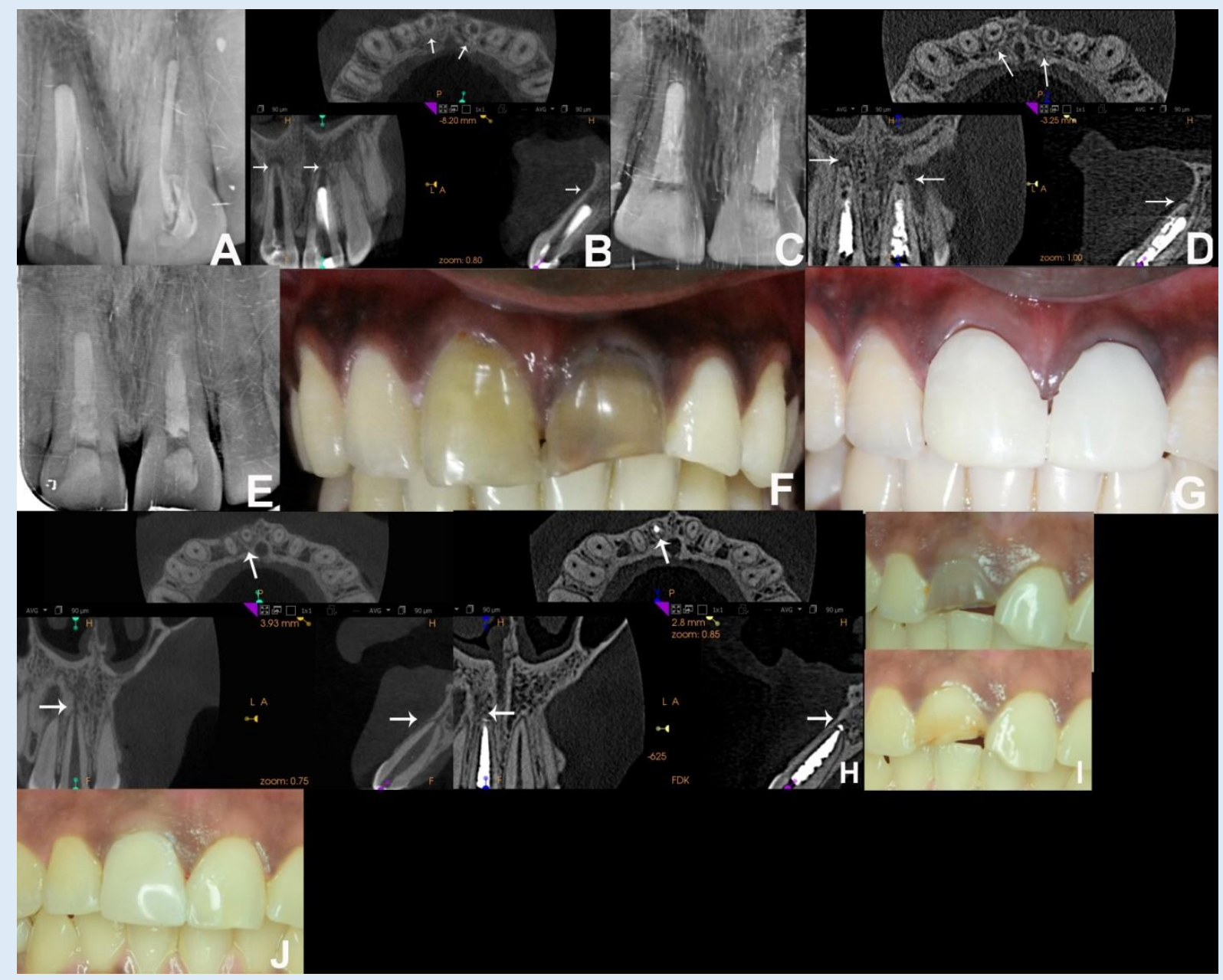

Figure 9: A, B: Pre-operative radiograph of root canal treated right and left maxillary central incisors with open apex (arrow) of a $20 \mathrm{yr}$-old male patient in year 2018. C: Immediate postobturated radiograph of maxillary central incisors with Biodentine apical plug and backfill with thermoplasticized gutta-percha. D, E: 13-months post-treatment CBCT and radiograph images displaying periapical healing (arrow). F, G: Discoloration due to triple-antibiotic

Sherwood AI | Volume 1; Issue 2 (2020) | JDHOR-1(2)-011 | Review Article

Citation: Sherwood AI, et al. Biodentine: A Unique Bio-Active Endodontic Material with Versatile

Uses. J Dental Health Oral Res. 2020;1(2):1-15. 
paste (TAP) in both the maxillary central incisors managed with lithium di silicate ceramic full coverage crowns. H: Pre-and 22-months post-treatment CBCT images of an $18 \mathrm{yr}$-old female patient with right maxillary central incisor displaying open apex (arrow) managed with Biodentine apexification in year 2018. I, J: Discoloration caused by TAP managed by three-sittings of intra-coronal bleaching completed with composite restoration in right maxillary central incisor.

\section{Healing of Periapical Pathosis When Biodentine is used as a Root Canal Sealer}

A 27 yr-old female patient with complaints of pain in her traumatized left maxillary central incisor reported to the department in 2017. Radiographic data, CBCT scan and clinical examination indicated that the tooth had a necrotic pulp with periapical lesion (Fig. 10). The tooth was managed by non-surgical root canal treatment with calcium hydroxide intra-canal medicament for 1-week, ultrasonic irrigation activation followed by thermoplastic obturation with Biodentine as root canal sealer. Sixteen months following root canal treatment the patient returned for assessment and a CBCT scan showed excellent periapical healing and resolution of the periradicular pathosis (Fig. 10).

A 23 yr-old male patient with complaint of pain in a mandibular left second molar tooth reported to the department in 2018. On clinical examination a deep caries with pulpal involvement and a non-draining extra-oral sinus tract was present near the angle of mandible. Radiographic and CBCT examination confirmed the pulpal involvement and a periapical radiolucency evidencing erosion of the lingual cortical plate (Fig. 10). Non-surgical root canal treatment was the preferred treatment using Bio-dentine. At twelve months a CBCT scan showed complete resolution of the periapical lesion (Fig. 10).

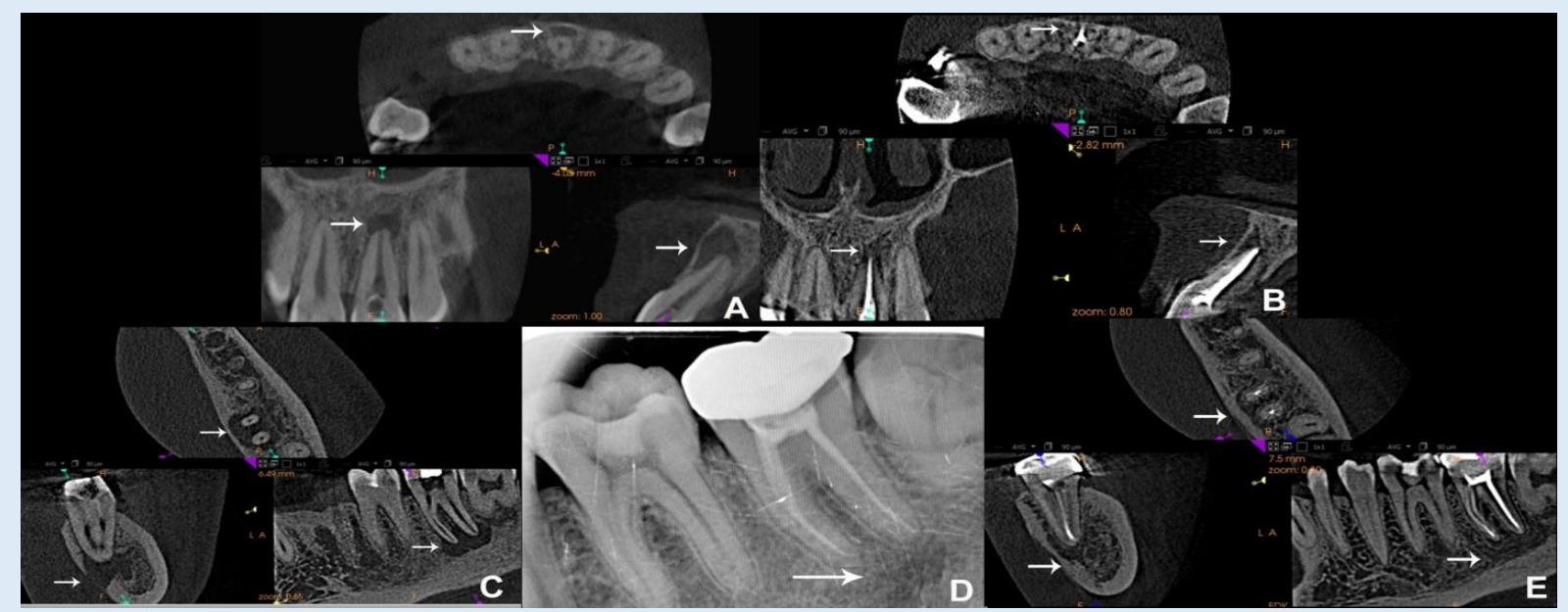

Figure 10: A: Pre-operative CBCT image of left maxillary central incisor with periapical pathosis (arrow). B: 16-months post-treatment CBCT scan exhibiting periapical resolution

Sherwood AI | Volume 1; Issue 2 (2020) | JDHOR-1(2)-011 | Review Article

Citation: Sherwood AI, et al. Biodentine: A Unique Bio-Active Endodontic Material with Versatile

Uses. J Dental Health Oral Res. 2020;1(2):1-15.

DOI: http://dx.doi.org/10.46889/JDHOR.2020.1206 
(arrow) of the lesion. C: Pre-operative CBCT of left mandibular second molar with periapical lesion (arrow) of pulpal origin involving lingual cortical plate. D, E: 12-months posttreatment radiograph and CBCT images showing periapical bone regeneration and healing (arrow).

\section{Surgical Management of Peripical Lesions of Pulpal-Origin with a Biodentine Apical Plug}

Periapical surgery with curettage with or without root-end resection was chosen for teeth exhibiting perapical radiolucencies with cortical plate involvement, as evidenced in CBCT scans. The teeth were obturated prior to surgery with a Biodentine apical plug and thermoplasticized gutta-percha backfill of the canal. The apical plug served two purposes:

1. Placement of this bioactive material in contact with periradicular tissues

2. If a root-end resection was done more surface area of Biodentine would be in contact with the periradicular tissues, thereby negating the need for a root-end filling

A 13 yr-old male patient was referred to the department in 2018 with complaints of pain and swelling in the mandibular anterior region (Fig. 11). On clinical examination both mandibular central incisors had necrotic pulps and right mandibular central incisor was rotated along with a lingually placed right mandibular lateral incisor (Fig. 11). The patient reported a history of trauma to his mandibular incisors two years prior. CBCT scan examination showed a large periapical radiolucency involving the two central incisors, along with labial cortical plate erosion (Fig. 11). To alleviate the abscess that was present, an incision and drainage was performed. Root canal treatment of the two mandibular central incisors was completed as per earlier mentioned protocol. Surgical treatment option was chosen using Bio-dentine; periapical curettage was performed without root-end resection (Fig. 11), and Platelet-Rich Fibrin (PRF) was placed as graft material in bony defect. Histopathological examination of the curetted tissue indicated a periapical granuloma. A 12 months post-treatment CBCT scan showed excellent periapical healing, with resolution of the periapical radiolucency and bone regeneration (Fig. 11).

A $21 \mathrm{yr}$-old female patient reported to department in 2019 with complaints of pain in root canal treated left maxillary central and lateral incisors that were restored with ceramic crowns (Fig. 11). Radiographic examination indicated that the previous root canal treatment was inadequate and a large periapical radiolucency involving the roots of the left maxillary central and lateral incisors was present (Fig. 11). CBCT scan examination showed the periapical radiolucency had eroded the labial cortical plate (Fig. 11). The ceramic crowns were removed followed by completion of root canal treatment with Biodentine apical plug obturation (Fig. 11). Root-end resection of both the right maxillary central and lateral incisors was performed along with a PRF graft material in the bony defect (Fig. 11). Histopathological examination showed

Sherwood AI | Volume 1; Issue 2 (2020) | JDHOR-1(2)-011 | Review Article

Citation: Sherwood AI, et al. Biodentine: A Unique Bio-Active Endodontic Material with Versatile

Uses. J Dental Health Oral Res. 2020;1(2):1-15. 
scattered areas of epithelial lining in the curetted tissue and a diagnosis of periapical granuloma with isolated cystic elements was given. Post-endodontic restorations with new lithium-disilicate full coverage crowns were placed (Fig 11). 12-months post-treatment CBCT scan showed excellent periapical healing and bone regeneration (Fig 11).

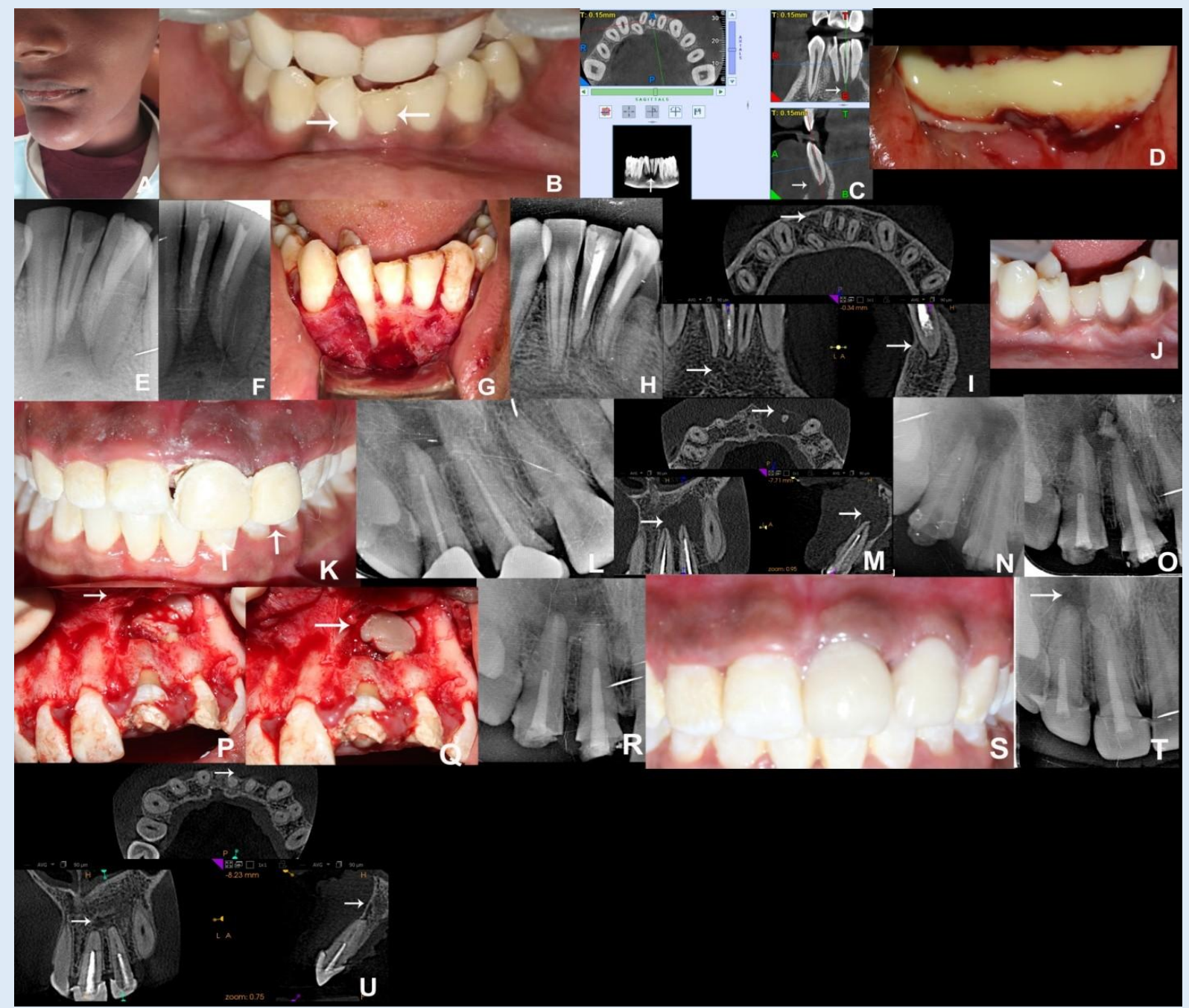

Figure 11: A, B and C: Pre-operative photograph and CBCT images of extra-oral swelling, discolored mandibular left central incisor and rotated right mandibular central incisor with periapical radiolucency eroding the labial cortical plate. D: Incision and drainage of periapical abscess. E and F: Pre- and immediate post-root canal treatment radiographs Biodentine placed in the apical 1/3rd of the canal. G: Periapical curettage and placement of Platelet-Rich Fibrin graft (PRF) in the bony defect. H, I and J: 12-months post-treatment periapical radiograph, CBCT and clinical images displaying periapical bone regeneration (arrow). K, L and M: Root canal treated left maxillary central and lateral incisor clinical (arrow), radiograph and CBCT images displaying periapical radiolucency eroding labial cortical plate (arrow). $\mathbf{N}$ and $\mathbf{O}$ : Gutta-percha removed and apical 1/3rd of root canal obturated with Biodentine remaining root canal obturated with thermoplasticized gutta- 
percha. P and Q: Periapical bone defect (arrow) placed with PRF graft material after root-end resection. R: Immediate post-surgical radiograph. S: New lithium di silicate full coverage crowns for left maxillary central and lateral incisors. T and $\mathbf{U}$ : 12-months periapical radiograph and $\mathrm{CBCT}$ images showing periapical healing and bone regeneration (arrow).

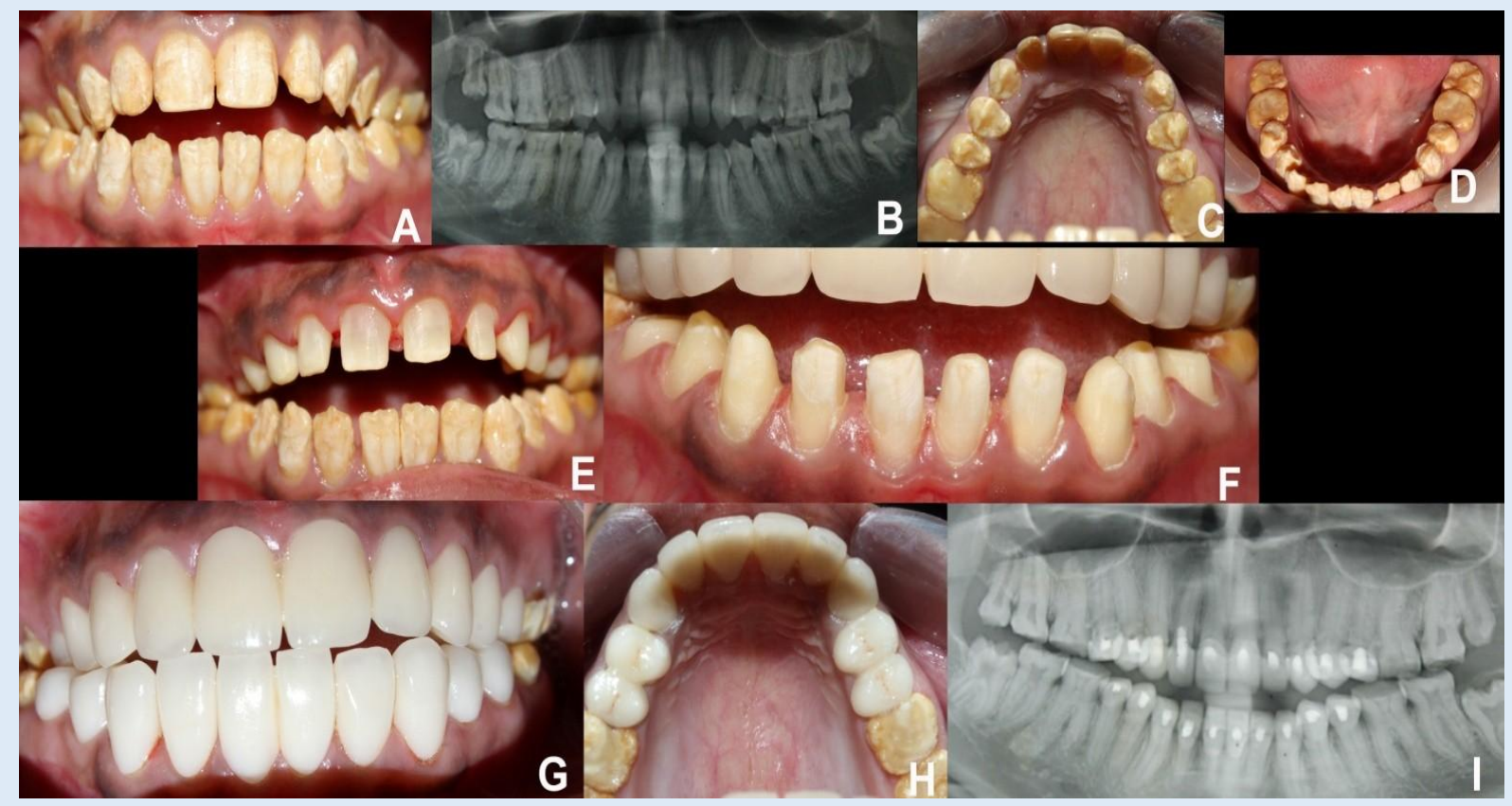

Figure 12: A, B, C and D: Pre-operative clinical and orthopantogram of patient. $\mathbf{E}$ and $\mathbf{F}$ : Full crown tooth preparation of the maxillary and mandibular teeth. $\mathbf{G}$ and $\mathbf{H}$ : Lithium di silicate full crown restorations for the maxillary and mandibular teeth. I: Post-treatment orthopantogram showing pulpotomy procedure for the full crown restored teeth.

\section{Intentional Pulpotomy in Amelogenesis Imperfecta Management}

A 17 yr-old female patient reported to the department for management on unaesthetic appearance of her teeth in the year 2019 (Fig. 12). Upon clinical examination she was diagnosed as having amelogenesis imperfecta. An orthopantogram showed no caries, pulpal or periapical pathosis (Fig. 12). She was advised to have metal-free lithium di silicate ceramic crowns for her maxillary and mandibular teeth. Crowns were delivered from left second premolar to right second premolar. Previous experience with Biodentine pulpotomies for sensitive, attrited molar teeth prompted us to have the teeth to be restored with crowns to have Biodentine pulpotomies prior to tooth preparation (Fig. 12) [5]. The rationale for choosing pulpotomy was to prevent pulpal damage during extensive tooth preparation required for metalfree ceramic crowns.

In addition to the cases presented here, Biodentine has been used in other clinical scenarios, such as the management of dens invaginatus [6]. 


\section{Conclusion}

Better understanding of progression of pulpal pathology and periapical healing has allowed for the use of bioactive materials, such as Bio-dentine, for more conservative treatment options, thereby avoiding extensive and invasive procedures in preserving the tooth and dentition.

\section{References}

1. Jefferies SR. Bioactive and biomimetic restorative materials: a comprehensive review. Part I. J Esthet Restor Dent. 2014;26:14-26.

2. Parirokh M, Torabinejad M, Dummer PMH. Mineral trioxide aggregate and other bioactive endodontic cements: an updated overview - part I: vital pulp therapy. Int Endod J. 2018;51:177-205.

3. Zhou HM, Shen Y, Wang ZJ, Li L, Zheng YF, Häkkinen L, et al. In-vitro cytotoxicity evaluation of a novel root repair material. J Endod. 2013;39(4):478-83.

4. Camilleri J, Sorrentinob F, Damidotc D. Investigation of the hydration and bioactivity of radiopacified tricalcium silicate cement, Biodentine and MTA Angelus. Dent Mater. 2013;29:580-93.

5. Subashri V, Sherwood IA, Gutmann JL, Vaanjay M, Prince EP. Evaluation of 3 different treatment modalities for conservative management of attrited sensitive molar teeth-A preliminary 12 week report. Aust Endod J. 2020.

6. Subashri V, Sherwood IA, Gutmann JL, Vaanjay M, Prince EP. Evaluation of 3 different treatment modalities for conservative management of attrited sensitive molar teeth-A preliminary 12 week report. Accepted for publication. Aust Endod J. 2020. 